\title{
Interference Measurement of Rough Surface Relief
}

\author{
Oleg V. Angelsky, Olexander P. Maksimyak, Peter P. Maksimyak \\ Correlation Optics Dept, Chernivtsy University \\ 2 Kotsyubinsky St., Chernivtsy 58012, Ukraine
}

The optical techniques are non-contact, non-destructive and highly efficient for diagnostics of rough surfaces. The optical techniques may be divided into profile interference and heterodyning techniques ${ }^{1,2}$, techniques based on measuring of the angular distribution of scattered radiation ${ }^{3}$, and optical correlation techniques, ${ }^{4}, 5$. High operation rates, data processing in the optical channel, high accuracy of measurements, feasibilities for real-time data processing, and small sizes of the measuring devices are the main advantages provided by optical correlation diagnostic methodology ${ }^{6}$. However, when the height of surface inhomogeneities exceeds the wavelength of the probing beam, and the specular component of the reflected radiation is absent, the unambiguous connection between the statistical parameters of the roughness and of the scattered field is lost. The new approaches of fractal and singular optics are consequently needed for diagnostics of such surfaces ${ }^{7,8}$. At the same time, these approaches so far provided only classification of rough surfaces into random and fractal ones.

In this paper, we promote the optical correlation technique for determining the height distribution function for surfaces with large surface inhomogeneities (roughness) and for the reconstruction of the relief structure of such a surface based on the results of measuring and processing of the longitudinal coherence function for the scattered field. For diagnostics of surfaces with large inhomogeneities one often uses the techniques of speckle optics ${ }^{4,9,10}$. In these cases, however, the statistics of the height distribution of the inhomogeneities influences the results of measurements, and the depth of roughness must not exceed 3 microns. Optimization of interference measurements and computer processing of them provide reconstruction of the relief of regular surfaces with resolution $0.5 \mathrm{~nm}$.

Illumination of a rough surface with the relief structure $h(x, y)$ by a normally incident monochromatic plane wave results in the spatial phase modulation of the reflected wave at the boundary field, $4 \pi h(x, y) / \lambda$. The longitudinal coherence function $\Gamma(z)$ of the scattered field is not assumed to be spatially restricted. If the same surface is illuminated by a polychromatic beam with a finite longitudinal coherence length, reflection from a rough surface is accompanied by spreading of the longitudinal coherence function of the resulting field ${ }^{5,6}$ due to various delays of the partial signals, which are determined by the roughness relief. As a result, the modulus of the complex degree of coherence of the field decreases.

We propose the following procedure for determining $\Gamma(z)$. In the Michelson interferometer, see Fig. 1, the monochromatic or a polychromatic field at the plane of the image of a rough surface formed by a microobjective is mixed with a monochromatic or a polychromatic reference field, respectively.

Subseguently, one determines the correlation of the monochromatic interference image of a rough surface with high visibility over the aperture with the set of polychromatic interference images with nonuniform visibility obtained with varying path differences in the interferometer. This algorithm provides determination of the longitudinal covariation function of the images and deriving the longitudinal coherence function of a polychromatic field scattered from the object, $\Gamma(z)$.

In our experiments, we use a solid-state laser with a wavelength $\lambda=532 \mathrm{~nm}$ and a coherence length 4 $\mathrm{cm}$ (considerably exceeding the maximal span of heights of the surface inhomogeneities) as the monochromatic source. In such a manner, we provide high visibility of an interference pattern over the observed area of a rough surface, see Fig. 2. This relief was constituted by two identical orthogonal sinusoidal gratings with the period $15 \mu \mathrm{m}$ and modulation depth $1 \mu \mathrm{m}$. 
The intensity of the resulting field at the interferometer output becomes

$$
I^{m}(x, y, z)=I_{0}^{m}+I_{s}^{m}(x, y)+2 \sqrt{I_{0}^{m} I_{s}^{m}(x, y)} \cos \left\{\frac{4 \pi}{\lambda}[h(x, y)-z]\right\},
$$

where $I_{0}^{m}$ is the reference wave intensity, and $I_{s}^{m}(x, y)$ is the intensity distribution at the surface image in monochromatic light.

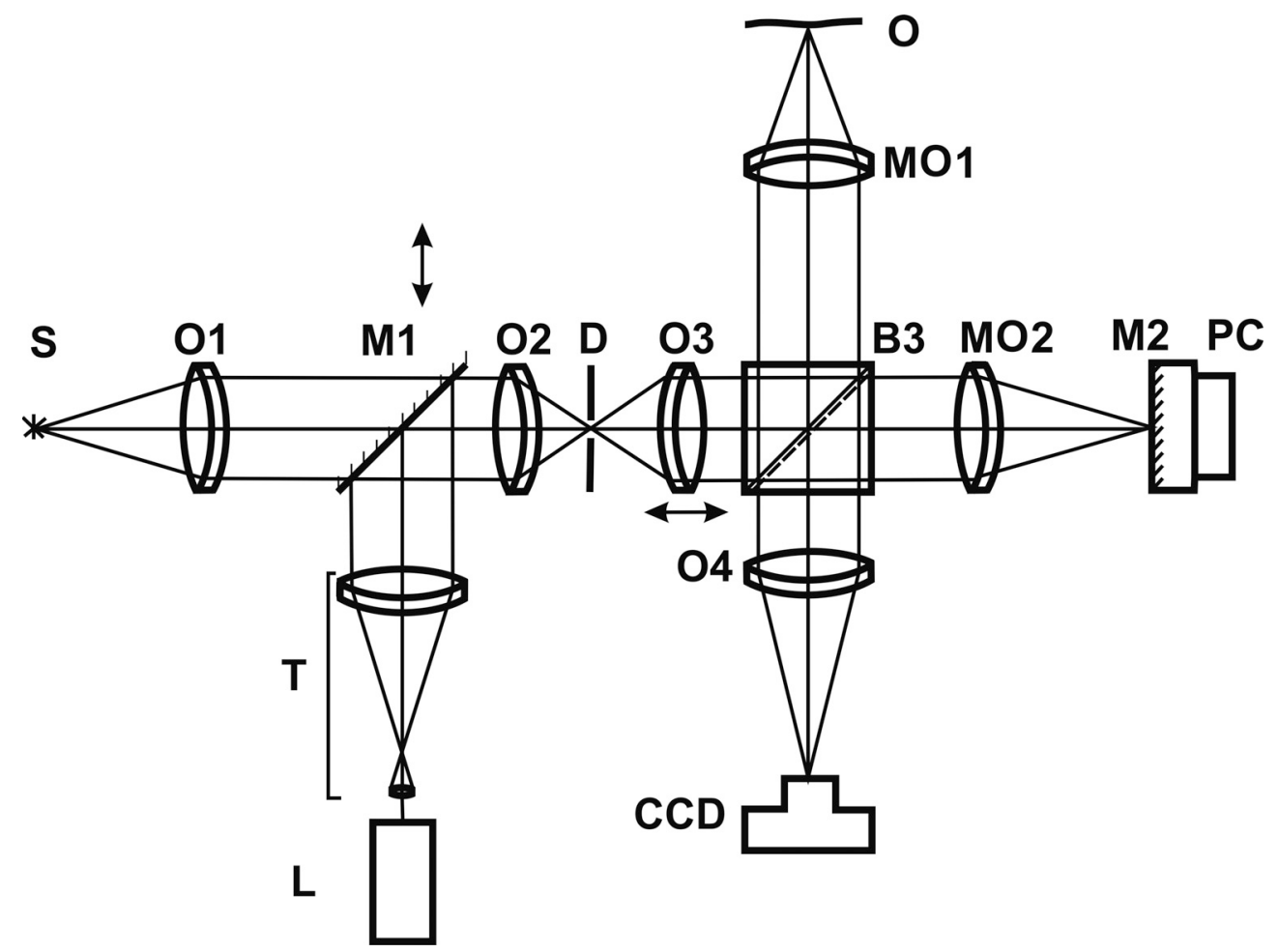

Fig. 1. Experimental arrangement: $\mathrm{S}$ - temporal source; $\mathrm{L}$ - solid-state laser; $\mathrm{O} 1, \mathrm{O} 2, \mathrm{O} 3$ and $\mathrm{O} 4$ objectives; T - telescope; M1- moving mirror, M2 - mirror; D - diaphragm; BS - beam-splitter; MO1 and MO2 - micro-objectives; O - surface of interest; PC - piezo-ceramics.

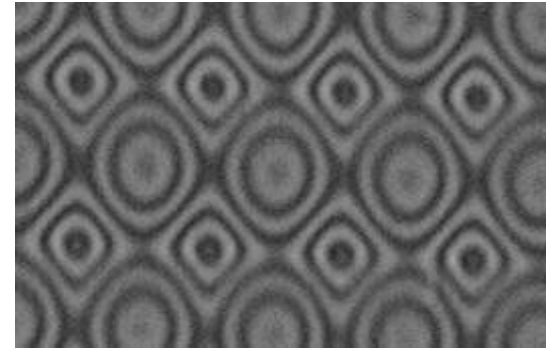

$\mathrm{Z}=0.1 \mu \mathrm{m}$

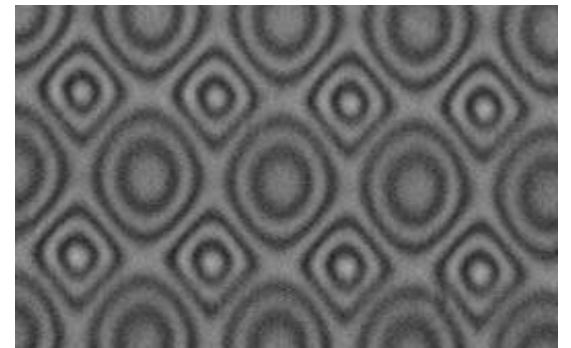

$\mathrm{Z}=0.5 \mu \mathrm{m}$

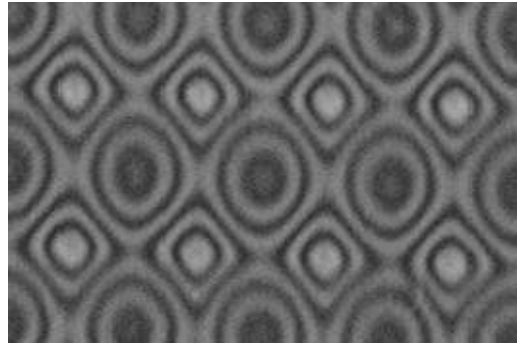

$\mathrm{Z}=0.9 \mu \mathrm{m}$

Fig. 2. The frames of a monochromatic flow for the surface with regular relief.

The use of a temporal source with a finite coherence length in the Michelson interferometer enables a longitudinal scanning of the rough surface relief, e.g. the step-by-step interference selection of crosssections of the relief, which are parallel to a mean surface line. Intensity distributions of the resulting interference fields are shown in Fig. 3 for various optical path differences in the interferometer legs.

The experimentally obtained resulting white-light interference pattern for the optical path difference $z$ is described by the following relation:

$$
I(x, y, z)=I_{0}+I_{s}(x, y)+2 \sqrt{I_{0} I_{s}(x, y)} \mid \Gamma\left(z-z_{0}\right) \cos \left\{\frac{4 \pi}{\lambda}[h(x, y)-z]\right\},
$$


where $I_{0}$ is the reference wave intensity, $I_{s}(x, y)$ is the intensity distribution at the surface image in polychromatic light, and $z_{0}$ is any starting position.

The mutual longitudinal intensity covariation function (MLICF) of $I^{m}(x, y, z)$ and $I(x, y, z)$ is determined by the following relation:

$$
B(z)=\left\langle I^{m}\left(x, y, z_{0}\right) l(x, y, z)\right\rangle .
$$

Averaging is carried out over the entire observed area of the rough surface.

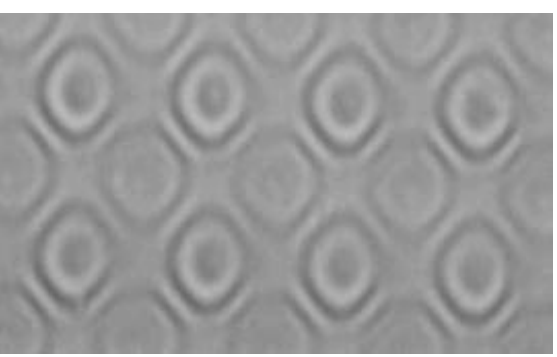

$\mathrm{Z}=0.1 \mu \mathrm{m}$

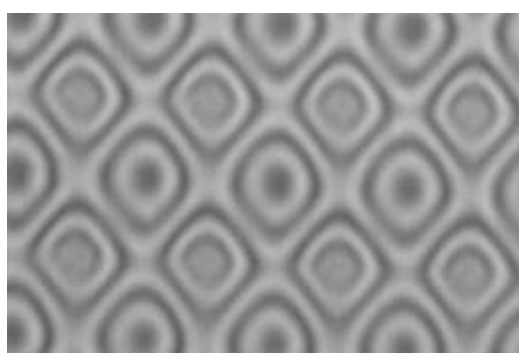

$\mathrm{Z}=0.5 \mu \mathrm{m}$

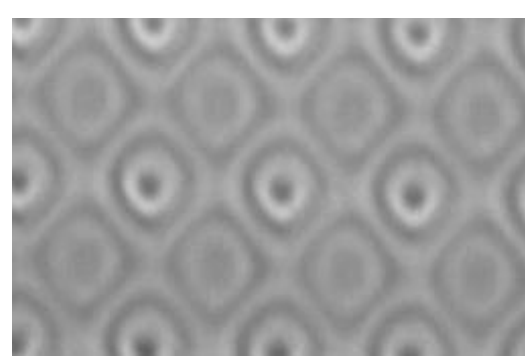

$\mathrm{Z}=0.9 \mu \mathrm{m}$

Fig. 3. The frames of a polychromatic flow for the surface with regular relief.

A typical form of the MLICF is shown in Fig. 4. The distance between the maxima is $\lambda / 2$ (266 nm, in our case), which is convenient for the use as the marking the coherence function with respect to the roughness height parameter.

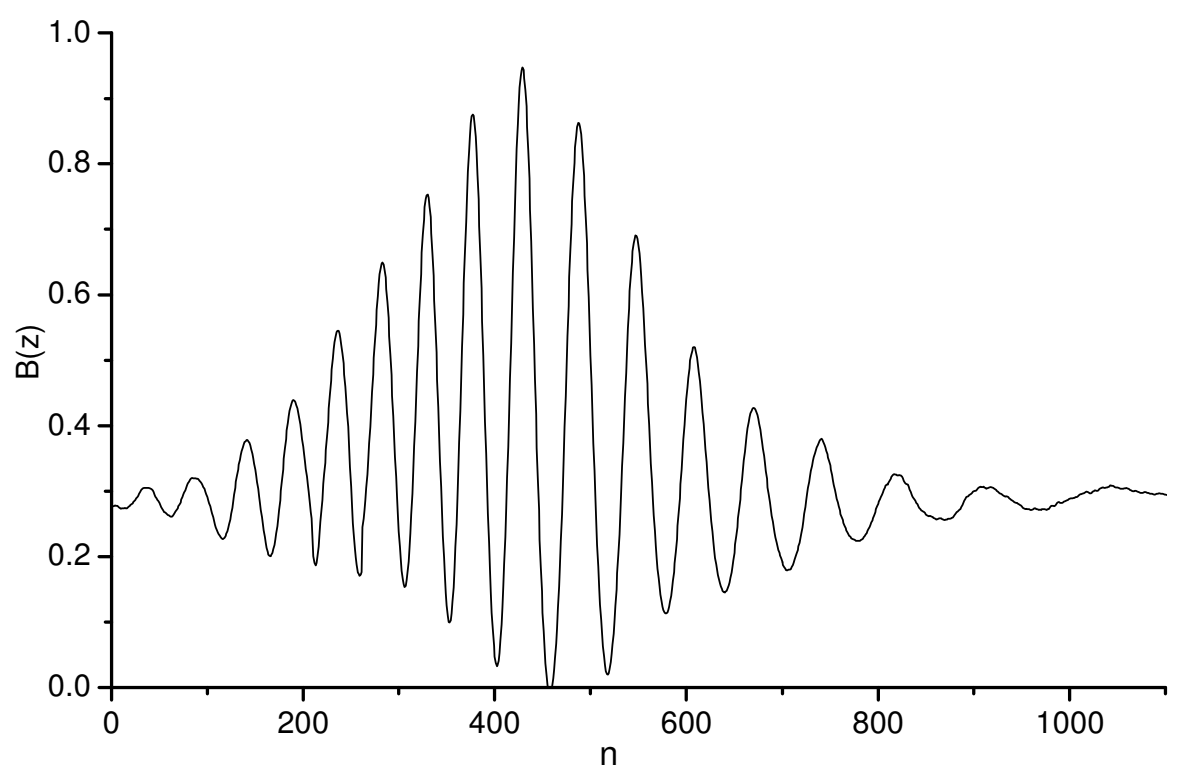

Fig. 4. Typical mutual longitudinal intensity covariation function (MLICF) of the monochromatic interference image of a rough surface and the polychromatic interference images for various optical path differences between the legs of interferometer ( $n$-number of frame).

Experimental studies have been carried out using the arrangement shown in Fig. 1. We used a Nd:YAG laser, L, operating at $532 \mathrm{~nm}$ and a temporal source, $\mathrm{S}$, as the sources of probing radiation. Radiation of the source $\mathrm{S}$ is projected onto the $20 \mu \mathrm{m}$-diam diaphragm, D, positioned at the focus of the objective $\mathrm{O} 3$ (focal length $200 \mathrm{~mm}$ ) by the objectives $\mathrm{O} 1$ and $\mathrm{O} 2$. In this way, we form a white-light beam with a degree of spatial (transverse) coherence of $95 \%$. If the mirror $M$ is used, the laser beam is spread using the 
telescope $T$ and focused on the same diaphragm $D$. The beamsplitter BS divides the incident beam into the reference and the object beam. The images of the surface of interest, $\mathrm{O}$, and the reference mirror, $\mathrm{M} 2$, are projected onto the CCD-camera by the micro-objectives MO1 and MO2, and the objective O4. The mirror M2 with the micro-objective MO2 is shifted along the direction of propagation of the beam, facilitating a change in the optical path delay of the interferometer legs. Optical phase difference (within a wavelength) is provided by a piezo-ceramic PC mounted with a mirror M2. By moving the surface of interest along the direction of propagation of the beam, one can obtain an image of the surface using a black and white CCD-camera.

We used a CCD-camera with a maximum spectral sensitivity at $532 \mathrm{~nm}$, and studied the roughness of grinded plates of mono-crystalline silicon. Initially, we record two video sequences. The fist of them reflects the evolution of the monochromatic interference field while changing the optical path difference between the object and the reference beam within one wave-length. The frames from this flow are shown in Fig. 2. The second sequence reflects the evolution of the white-light interference field for changed optical path delays within the maximal depth of the rough surface relief. The frames are shown in Fig. 3. Then, one frame of the monochromatic interference field, is correlated with each frame of the polychromatic interference field.

Further, from the maximal magnitude $B_{\max }$ of the cross-correlation function:

$$
B(n)=\frac{\sum_{i=1}^{N} \sum_{j=1}^{M}\left[I_{i j, 0} * I_{i j}^{m}(n)\right]}{\left(\sum_{i=1}^{N} \sum_{j=1}^{M} I_{i j, 0}\right) *\left(\sum_{i=1}^{N} \sum_{j=1}^{M} I_{i j}^{m}(n)\right)}
$$

one selects the frame $I_{i j, 0}^{m}$ of the monochromatic flow, which strongly correlates with the frame $I_{i j, 0}$ of the polychromatic flow and for which interference fringes of the minimal intensity pass through all peaks of a surface simultaneously (Fig. 3 c).

The frame $I_{i j, 0}^{m}=I_{i j}^{m}(k)$ is the initial one for the procedure of reconstruction of the regular surface relief. That is why, one re-numerates the frames of the monochromatic flow:

$$
I_{i j}^{m}(I)=I_{i j}^{m}(n-k) .
$$

The monochromatic flow $I_{i j}^{m}(I)$ includes many cross-sections of the relief, which are determined by the coordinate distributions of the minimal magnitudes of intensity at the frame. It is necessary to select the coordinates of the relief corresponding to its heights. For this purpose, one uses the coordinate distribution of the correlation of the monochromatic flow's initial frame, $I_{i j}^{m}(I)$, with the frame $I_{i j, 0}$ of the polychromatic flow. Namely, from the frame $I_{i j, 0}$ one forms the binary frame,

$$
I_{i j, 0}^{B}=\left\{\begin{array}{lll}
1, & \text { for } & I_{i j, 0}^{n} \geq C \\
0, & \text { for } & I_{i j, 0}^{n}<C
\end{array},\right.
$$

where the normalized in intensity frame is determined as $I_{i j, 0}^{n}=\frac{I_{i j, 0}-I_{i j, 0}^{\min }}{I_{i j, 0}^{\max }-I_{i j, 0}^{\min }}$, by normalizing of the intensity distribution on the maximal and minimal intensities within the frame and by comparing of intensity with some level $c$.

Further, one finds out the distribution of binary correlation of the initial frame of the monochromatic flow with the binary frame $I_{i j, 0}^{B}$ of the polychromatic flow:

$$
B_{i j}^{B}(0)=\left\{\begin{array}{lll}
1, & \text { for } & B_{i j}^{n}(0) \geq d \\
0, & \text { for } & B_{i j}^{n}(0)<d
\end{array},\right.
$$


where $B_{i j}^{n}(0)=\frac{B_{i j}(0)-B_{i j}^{\min }(0)}{B_{i j}^{\max }(0)-B_{i j}^{\min }(0)}$ is the normalized correlation function; $B_{i j}(0)=I_{i j}^{m}(0) * I_{i j, 0}^{B} ; d$ the level of binarization of the correlation function.

To control of the width of the scanning band, we introduce the procedure of Gaussian averaging with dispersion $\sigma$ of the binary correlation function followed by its averaging and binarization at the level $e$ :

$$
\begin{gathered}
B_{i j}^{G}(0)=\sum_{f=-3 \sigma}^{3 \sigma} \sum_{g=-3 \sigma}^{3 \sigma} B_{i+f, j+g}^{B}(0) \frac{1}{2 \pi \sigma^{2}} \exp \left(-\frac{f^{2}+g^{2}}{2 \sigma^{2}}\right) ; \\
B_{i j}^{B G}(0)=\left\{\begin{array}{lll}
1, & \text { for } & B_{i j}^{n G}(0) \geq e \\
0, & \text { for } & B_{i j}^{n G}(0)<e
\end{array}\right.
\end{gathered}
$$

where $B_{i j}^{n G}(0)=\frac{B_{i j}^{G}(0)-B_{i j}^{G \min }(0)}{B_{i j}^{G \max }(0)-B_{i j}^{G \min }(0)}$ is the normalized correlation function resulting from Gaussian averaging.

Let us determine the distribution of the following binary correlation $B_{i j}^{B G}(I)$ obtained as the correlation of $B_{i j}^{B G}(I-1)$ with the following frame $I_{i j}^{m}(I)$ of the monochromatic flow. For the each frame, the procedures following Eqs. (6) - (9) are repeated. The coefficients $c, d$ and $e$ are chosen to be the same for all steps and to provide optimal scanning of the relief as a whole. The coordinates of the each distribution of the binary correlation $B_{i j}^{B G}(I)$ are associated with the magnitude of the relief height in correspondence with the frame number. For that, this magnitude can be associated with any point once only. The coordinates and the magnitudes of the frame numbers are accumulated as the single massive, where the data on the surface relief is are formed. Then, the obtained massive is scaled on the crosscorrelation function of the intensity distribution for the initial frame of the monochromatic flow $I_{i j}^{m}(0)$ and intensity distribution of the monochromatic flow:

$$
B(I)=\frac{\sum_{i=1}^{N} \sum_{j=1}^{M}\left[I_{i j}^{m}(0) * I_{i j}^{m}(I)\right]}{\left(\sum_{i=1}^{N} \sum_{j=1}^{M} I_{i j}^{m}(0)\right) *\left(\sum_{i=1}^{N} \sum_{j=1}^{M} I_{i j}^{m}(I)\right)} .
$$

The distribution $B(I)$ is of cosinusoidal form, whose period constitutes some number of the frames, $k$, being equal to $\lambda / 2(266 \mathrm{~nm})$. Then, the relation among the frame number, $l$, and the height, $h$, will be $h=\lambda l / 2 k$. This relation permits to estimate a resolving power of the technique. So, for $k=500$ frames one has: $\delta h=\lambda / 2 k=0.532 \mathrm{~nm}$. Piezoelectric scanning system provides this resolving power.

Experimentally obtained relief of the studied surface is shown in Fig. 5.

The represented technique is applicable for diagnostics of one-dimensional and two-dimensional matrices and isolated lenses (spherical, cylindrical, non-spherical), prisms and other optical elements. 


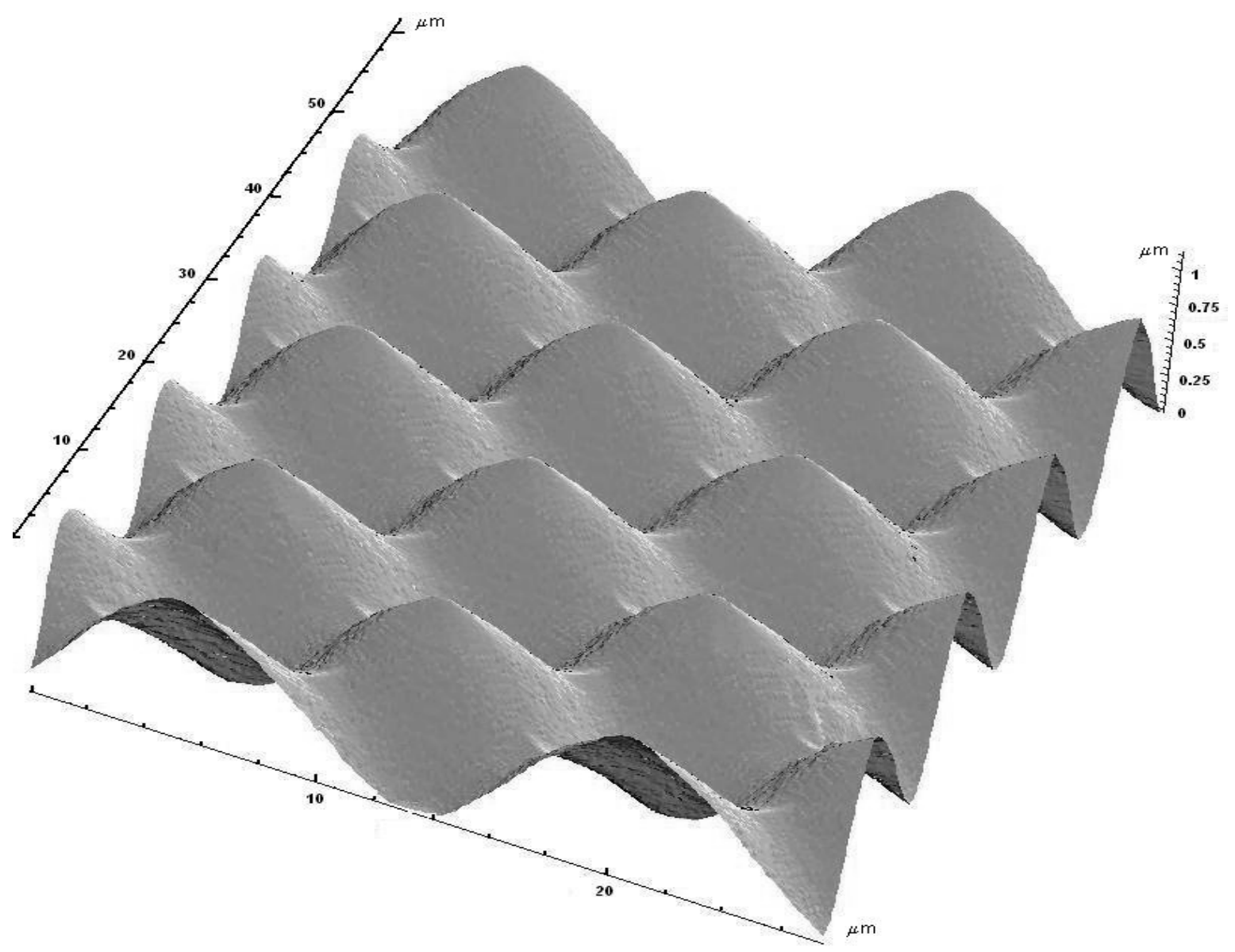

Fig. 5. Experimentally obtained relief of the surface with regular relief.

\section{REFERENCES}

1. J.M. Bennett and L. Mattson, "Introduction to Surface Roughness and Scattering", - Washington, D.C: Optical Society of America, (1999).

2. D. Pantzer, J. Politch, and L. Ek, "Heterodyne profiling instrument for the angstrom region," Appl. Opt., 25, pp.4168-4172 (1986).

3. P. Beckmann and A. Spizzichino, "The Scattering of Electromagnetic Waves from Rough Surfaces", - , London: Pergamon Press, (1963).

4. R.S.Sirohi (Ed), "Speckle Metrology", (New York: Marcel Deker, (1993).

5. O. V Angelsky., S. G. Hanson P. P. Maksimyak, "The Use of Optical-Correlation Techniques for Characterizing Scattering Object and Media". - Bellingham: SPIE Press PM71, (1999).

6. O.V. Angelsky, P.P. Maksimyak. Optical Correlation Diagnostics of Surface Roughness In: Handbook of Coherent Domain Optical Methods. Biomedical Diagnostics, Environmental and Material Science, ed. by V.V. Tuchin (Boston, Kluwer Academic Publishers), V.1, 43-92 (2004).

7. O.V.Angelsky, D.N.Burkovets, P.P.Maksimyak, S.G. Hanson "Applicability of the singular-optics concept for diagnostics of random and fractal rough surfaces", Applied Optics, 42, 4529-4540 (2003).

8. O.V. Angelsky, S. G. Hanson, A. P. Maksimyak and P. P. Maksimyak, "Interference diagnostics of white-light vortices" Opt. Express 13, 8179-8183 (2005).

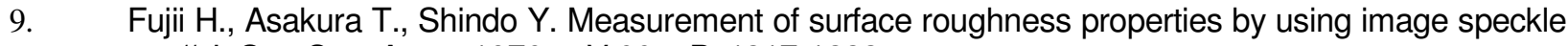
contrast // J. Opt. Soc. Am. - 1976. - V.66. - P. 1217-1222.

10. Spraque R. A. Surface roughness measurement using white light speckle // Appl. Opt. - 1972. - V.11. - P. 2811-2819. 